\title{
Shallow Implantation of "Size-Selected" Ag Clusters into Graphite
}

\author{
S. J. Carroll, P. D. Nellist, and R.E. Palmer \\ Nanoscale Physics Research Laboratory, School of Physics and Astronomy, The University of Birmingham, \\ Edgbaston, Birmingham B15 2TT, United Kingdom \\ S. Hobday and R. Smith \\ School of Mathematics and Physics,Loughborough University, Loughborough, Leicestershire, LE11 3TU, United Kingdom
}

(Received 22 July 1999)

\begin{abstract}
We have investigated the implantation of $\operatorname{Ag}_{N}(N=20-200)$ clusters into a graphite substrate over the range of energies $(E) 0.75-6 \mathrm{keV}$ using molecular dynamics simulations. We find that after implantation the silver clusters remain coherent, albeit amorphous, and rest at the bottom of an open tunnel in the graphite created by the impact. It is found that the implantation depth of the clusters varies linearly as $E / N^{2 / 3}$. We conclude that the cluster is decelerated by a constant force proportional to its cross-sectional area. We also identify a threshold energy for surface penetration associated with elastic compression of the graphite substrate.
\end{abstract}

PACS numbers: 79.20.Rf, 61.46.+w, 68.55.Ln, 81.05.Ys

There has been great interest in the physics of small clusters arising from the discovery that their electronic [1], chemical [2], magnetic [3], and thermodynamic [4,5] properties are size dependent. Technological applications require clusters to be deposited on surfaces, and so the physics of the deposition process is becoming increasingly important [6-10]. The ability to create a monodispersed array of size-selected clusters by deposition is thus an important goal. For example, systems of monodispersed clusters have been shown to display cluster-size dependent catalytic activity $[11,12]$. Size-selecting cluster sources can deposit clusters with a desired size $(N)$ ) and energy $(E)[13,14]$. In order to produce monodispersed arrays, however, the diffusion [8-10] and aggregation of the clusters on the surface has to be prevented. In this Letter we use molecular dynamics (MD) simulations to investigate the energetic deposition of silver clusters, containing 20-200 atoms, onto graphite. We find that, for high enough energies, the clusters implant into the graphite, and remain immobile at the bottom of a short open tunnel. This suggests intriguing catalytic behavior. The clusters implant via a mechanism of penetrating successive graphite planes immediately beneath the impact zone. We explore quantitatively the dependence of the implantation depth $(D)$ on the initial kinetic energy and size of the cluster, and find a relatively simple relationship (depth, $D \propto E / N^{2 / 3}$ ) from which it is possible to deduce the physics of the implantation process.

Previous work using MD simulations of cluster deposition on surfaces has usually involved wholly metallic or wholly covalent systems. In these systems such effects as intact (or "soft") landing of clusters through a breaking layer [15] (later realized experimentally [16]), flattening and surface mixing [17-21] of impacting clusters, as well as impact alloying and cratering [20-23], have been observed. However, mixed systems, such as silver clusters on graphite, are more likely to have important practical applications. The graphite substrate is a model in the field of cluster physics $[16,24-26]$ and is also an important support material in catalysis [27].

In this study, therefore, the molecular dynamics simulations not only have to connect two quite different types of (empirical) potentials describing the metal cluster and the covalently bonded graphite substrate, they must also describe the higher energy interactions between the different atomic species, and this is especially important for the energetic cluster impact investigated in this Letter. We use a set of potentials previously shown to reproduce closely experimental results [26]. The bonding in graphite was described using the $N$-body Brenner [28] potential, which gives the correct cohesive energy for carbon in the graphite and diamond structures. We simulated the Ag-Ag potential in the cluster using the many-body potential obtained via the embedded atom method by Ackland et al. [29]. For the bonding interaction between the C and $\mathrm{Ag}$ atoms we employed the empirical two-body potential obtained by Rafii-Tabar et al. [30] which produces an accurate simulation of STM results for the adsorption of $\mathrm{Ag}$ atoms and clusters on the graphite surface, and good agreement with experiment for small silver cluster deposition [26]. The repulsive walls of all of these potentials are smoothly joined at higher energies to the universal ZieglerBiersack-Littmark (ZBL) potential [31] which has been fitted to a database of single atom implantation data and is used as a standard for simulation of single ion implantation profiles. This splining process has been successfully used to predict the implantation profiles of boron and boron clusters [32] in silicon and in sputtering simulations [33]. Although the pairwise interaction of the bonding part of the Ag-C potential was fitted to surface adsorption, and thus cannot be expected accurately to describe the precise final bonding arrangements of $\mathrm{Ag}$ in $\mathrm{C}$, the higher energy nuclear interactions and hence the range of $\mathrm{Ag}$ in $\mathrm{C}$ (i.e., the implantation depth of the clusters, which is the focus 
of this work) should be adequately described by the ZBL repulsive wall.

The initial atomic arrangement within the clusters was a minimum energy structure obtained using a genetic algorithm [34]. The icosohedral structure predicted by this algorithm for $\mathrm{Ag}_{147}$ can be seen in Fig. 1(a). Simulations were performed using a graphite lattice up to the size $20 \times 20 \times 7 \mathrm{~nm}$, chosen to be large enough that the boundaries do not affect the resulting postimpact morphology. Damping was also introduced at the boundaries to prevent lattice displacement waves reflecting back into the impact zone [35]. The simulations ran for up to $20 \mathrm{ps,} \mathrm{with}$ a time step of $0.75 \mathrm{fs}$.

We describe a typical impact event in detail: the implantation into graphite of an $\mathrm{Ag}_{147}$ cluster traveling with $5.5 \mathrm{keV}$ kinetic energy (or $8 \mathrm{~km} \mathrm{~s}^{-1}, 18000 \mathrm{mph}$ ) shown
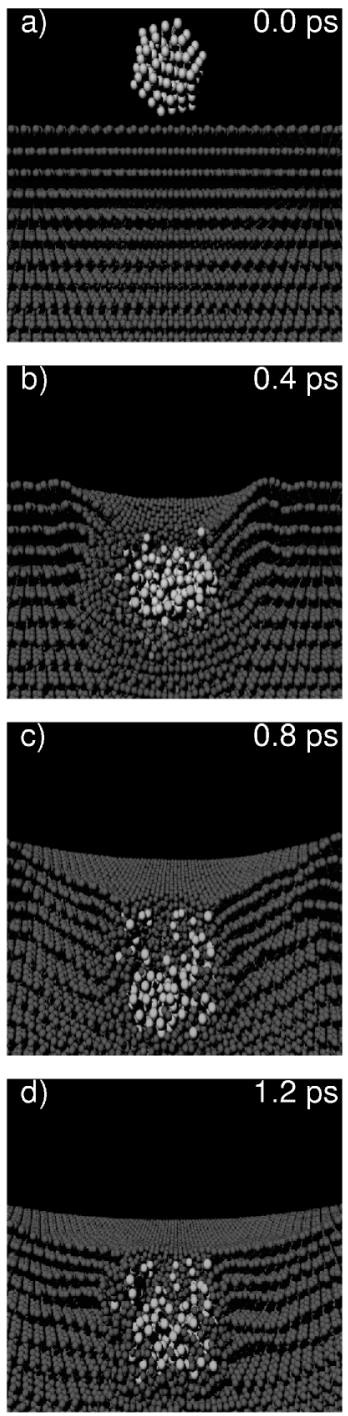

FIG. 1. Snapshots of the impact of a $\mathrm{Ag}_{147}$ cluster into graphite at $5.5 \mathrm{keV}$, viewed in cross section, as a function of time. $\mathrm{Ag}$ atoms are shown in light grey and $\mathrm{C}$ atoms are shown in dark grey. in Fig. 1. As the collision progresses we observe the cluster deforming, becoming amorphous (b), and penetrating into the graphite (c), which becomes locally depressed about the impact point. As the cluster proceeds the strain increases in the graphite until the $\mathrm{C}-\mathrm{C}$ bonds begin to break, after which the cluster begins to become embedded. After the cluster's impact energy is spent, the depression in the graphite begins to relax (d), after which the surface returns to the original level and the elastic compression energy is transported away as lattice vibrations. During the impact, as can be seen in Figs. 1(b) and 1(c), an amorphous region of carbon atoms in the immediate vicinity of the cluster is created. This is due to the high energy transfer from the cluster, possibly leading to local melting. At the end of the impact (d) we are left with a disordered cluster at the bottom of a short tunnel into the graphite, the tunnel walls and floor being composed of a thin layer of amorphous carbon.

In order to investigate the dependence of the implantation depth dependence on cluster energy and size, a whole series of impacts for clusters ranging in size between 20 and 200 atoms, and impact energies between 0.75 and $6.0 \mathrm{keV}$, were investigated. For each case, after sufficient time for the implanted cluster/graphite system to relax, the implantation depth $D$ was determined. The depth $D$ was measured by calculating the difference in height between the center of mass of the implanted cluster and the center of mass of a "halo" of the surface layer about the initial impact point. Some impacted clusters and their "halos" are presented in Fig. 2. In a manner similar to the $\mathrm{Ag}_{147}$ cluster impact presented in Fig. 1, the result of the $\mathrm{Ag}_{50}$ and $\mathrm{Ag}_{100}$ cluster impacts in Fig. 2 is a relatively coherent region of cluster material implanted beneath the graphite surface. As the impact energy of the clusters is increased [see Figs. 2(a)-2(c) or 2(d)-2(f)], the implantation depth increases, but the larger $\mathrm{Ag}_{100}$ cluster does not implant as
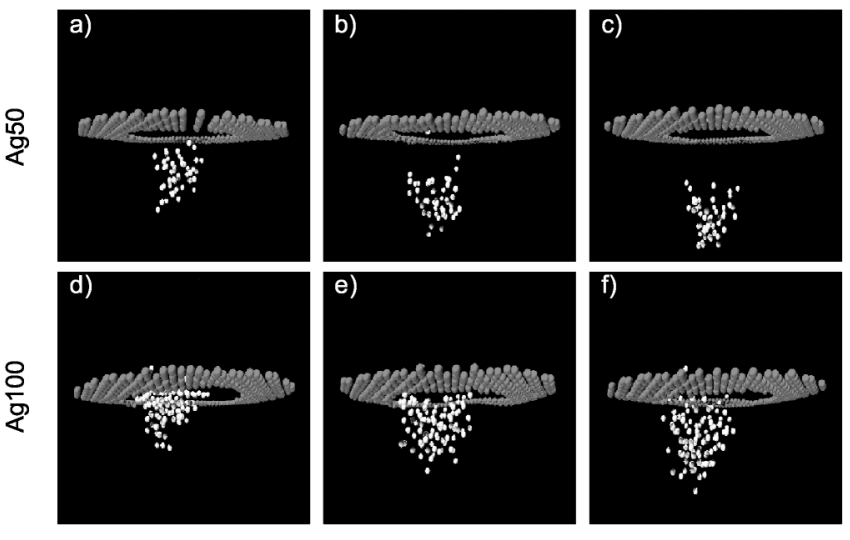

$2.0 \mathrm{keV}$

$2.5 \mathrm{keV}$

$3.0 \mathrm{keV}$

FIG. 2. Structures resulting from the impact of $\mathrm{Ag}_{50}$ and $\mathrm{Ag}_{100}$ clusters into graphite at $2.0,2.5$, and $3.0 \mathrm{keV}$. Clusters are shown with halos of the surface atoms used to determine implantation depth. (Other substrate atoms not shown for clarity.) 
deeply as the smaller $\mathrm{Ag}_{50}$ cluster, for the same impact energy. This reduced depth for a larger cluster is similar to the case of a macroscopic bullet: when the bullet is blunt, the penetration into the target is more shallow. In these initial observations it is clear that the cluster impact and resulting morphology of the impact zone are characterized by the impact energy $E$ and the number of atoms in the cluster, or cluster size $N$.

The dependence of the implantation depth on impact energy is presented for various cluster sizes in Fig. 3. We see that the implantation depth is linearly dependent upon the impact energy, but also that the gradients are affected by the cluster size $N$. The linear dependence of $(D \propto E)$ can be explained by Newtonian mechanics, if we suppose that the cluster is subjected to a constant resistive force (deceleration) as it is impeded by the graphite. Elastic deformation of the graphite crystal is not the dominant mechanism by which the cluster loses energy during implantation, since elastic losses would imply that $D \propto E^{1 / 2}$ (the resistive force is then proportional to the displacement of the graphite layers). Indeed, all of the graphite atoms would relax again after the impact. Further impacts at lower energies (where the clusters do not become fully implanted, but where a mixture of semi-implantation and flattening occurs) deviate from the straight line fits in Fig. 3 by curving toward the origin (not shown).

Figure 4 shows a plot of the gradients of the straight lines in Fig. 3 versus the cluster size $N$. As a guide to the eye, best fits are shown in Fig. 4 for the functional forms $k N^{-1}$ $\left(\sim\right.$ cluster volume $\left.{ }^{-1}\right), k N^{-2 / 3}\left(\sim\right.$ cross-sectional area $\left.{ }^{-1}\right)$, and $k N^{-1 / 3}$ ( $\sim$ cluster radius ${ }^{-1}$ ), where $k$ is the fitting constant in each case. Immediately obvious from Fig. 4 is that the data most closely resemble the $N^{-2 / 3}$ scaling. Indeed a $\log -\log$ plot of the data reveals scaling as $D \propto N^{-0.6 \pm 0.04}$. A simple model to explain the $E / N^{2 / 3}$ scaling of the implantation depth can be proposed by considering the number of bonds the cluster has to break to progress through the graphite. In this model, the number of bonds broken by the

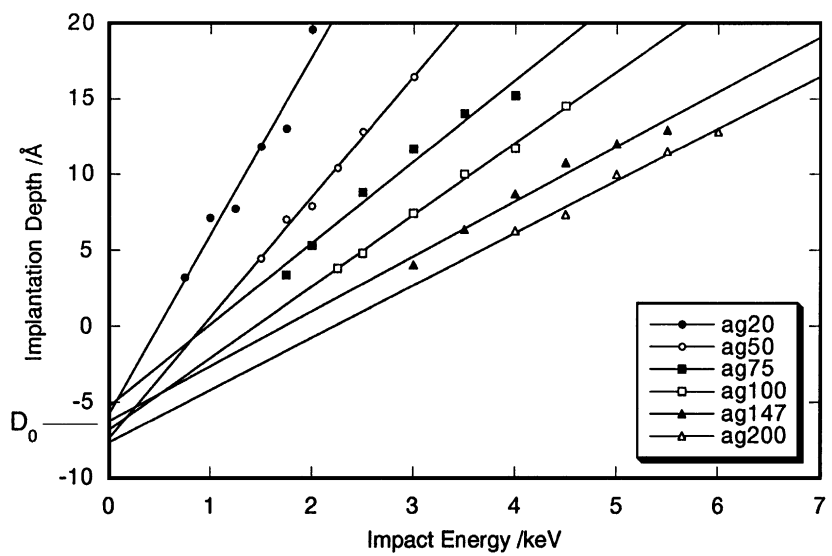

FIG. 3. Dependence of implantation depth $D$ on impact energy $E$ for different cluster sizes (inset). The corresponding best straight lines are also shown. cluster, and hence the energy expended, would be dependent on the volume of the tunnel which the cluster drives into the graphite. The energy expended before the cluster comes to rest (i.e., the impact energy $E$ ) would then be linearly dependent on the product of the implantation depth and the cross-sectional area of the cluster (which is proportional to $N^{2 / 3}$ ). Thus the implantation depth $D$ would be linearly dependent on the ratio $D \sim E / N^{2 / 3}$, which is indeed the case. (We also note that $E / N^{2 / 3}$ corresponds to the kinetic energy per unit area.) The value of -0.6 for the exponent, slightly smaller than the expected value of $-2 / 3$, is consistent with a slightly "reduced cross section" of the cluster. A small number of the outermost atoms on the equator of the cluster can be stripped off in the collision, effectively reducing the cross-sectional area of the tunnel.

From the fit, the energy given to the graphite by the impinging cluster is derived as $1.5 \mathrm{eV} / \mathrm{C}$ atom. To break all of the $\mathrm{C}-\mathrm{C}$ bonds in the tunnel carved out by the cluster, this energy should be $\sim 4.2 \mathrm{eV} / \mathrm{C}$ atom (assuming each carbon atom receives half of the $8.5 \mathrm{eV}$ required to break $3 \mathrm{C}-\mathrm{C}$ bonds) which is much larger than the measured energy, showing that the cluster does not vaporize the graphite beneath it. So the energy given to each $\mathrm{C}$ atom, as obtained from the fit, shows that the majority of the cluster's energy is spent breaking many, but not all, of the $\mathrm{C}-\mathrm{C}$ bonds in front of the cluster as it implants into the graphite.

We note also that in Fig. 3 the extrapolated linear fits for each cluster all cross the depth $D$ axis (where $E=0$ ) at a similar position, labeled $D_{0}$. This means that there is a minimum threshold energy for the clusters to penetrate the graphite surface. We associate this behavior with energy transfer to the substrate before the breaking of bonds. It is clear from the simulation, as evident in Fig. 1(b), that an elastic wave is propagated into the graphite substrate beneath the cluster. The energy given to this compressional wave could be described as $k_{c} N^{2 / 3}$, where $k_{c}$ is the energy per unit area required to compress the graphite beneath the

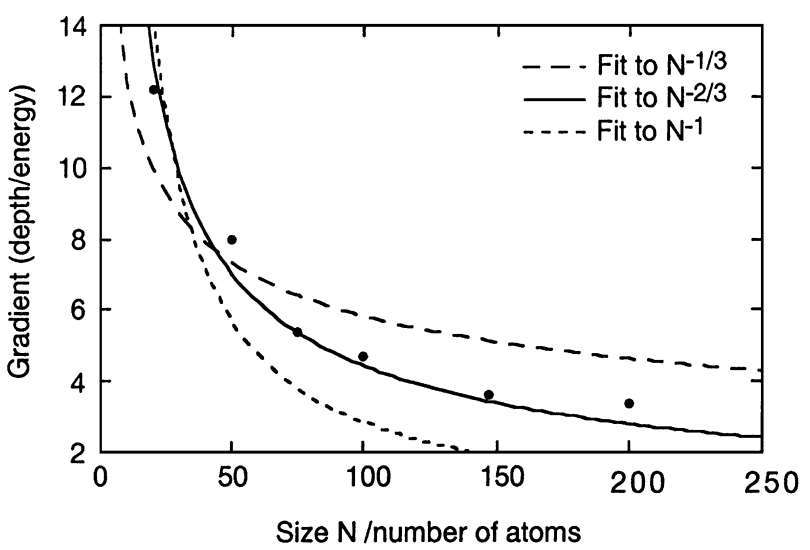

FIG. 4. Dependence of the ratio $D / E$ (i.e., the gradient in Fig. 3) on the cluster size $N$. Three different fits are also shown (see inset). 
cluster before $\mathrm{C}-\mathrm{C}$ bonds begin to break. By considering the energy balance of the system, we see that

$$
E=\frac{D N^{2 / 3}}{k}+k_{c} N^{2 / 3},
$$

where $k$ is the constant for the fit shown in Fig. 4. Rearranging for $D$, we see that the implantation is characterized by the equation

$$
D=\frac{k E}{N^{2 / 3}}-D_{0}
$$

Here $D_{0}=k k_{c}$, corresponding to the constant $D_{0}$ labeled in Fig. 3. Of course, in an experimental situation there will also be other channels for energy loss as the cluster approaches the surface, especially if it is charged, such as the creation of electron-hole pairs in the substrate.

The $E / N^{2 / 3}$ scaling identified should describe other cluster-surface systems, not just $\mathrm{Ag}_{N} /$ graphite, especially when the substrate is layered. Exceptions to this behavior are likely to arise in cases where full implantation does not occur, e.g., with a very "hard" substrate. Also, when the impact energy of the cluster is not sufficient for full implantation, the initial impact of the lowest atoms alone might dominate the impact. As shown previously [26], when the clusters are small enough to be similar in size to a surface unit cell, the behavior is strongly affected by their specific impact parameters, and in this case $E / N^{2 / 3}$ scaling would again break down.

In summary, we see that the silver clusters remain as coherent regions of silver, albeit amorphous, at the bottom of open tunnels into the graphite. The implantation depth $D$ varies linearly as $E / N^{2 / 3}$. In addition, the cluster has to overcome an energy threshold associated with compression of the graphite in order to become implanted. This knowledge should assist in the controlled production of nanostructures, such as novel catalytic structures created from monodispersed arrays of size-selected clusters.

This work was supported by the EPSRC and BNFL. S. J. C. would like to thank the University of Birmingham and the EPSRC for support. P.D.N. also acknowledges financial support from the Royal Society.

[1] W. Knight et al., Phys. Rev. Lett. 52, 2141 (1984).

[2] R. Whetten, D. Cox, D. Trevor, and A. Kaldor, Phys. Rev. Lett. 54, 1494 (1985).

[3] I. Billas, A. Chatelain, and W. de Heer, Science 265, 1682 (1994).

[4] C. Cleveland, W. Luedtke, and U. Landman, Phys. Rev. Lett. 81, 2036 (1998).
[5] M. Schmidt et al., Phys. Rev. Lett. 79, 99 (1997).

[6] M. Watanabe, T. Miyazaki, and T. Kanayama, Phys. Rev. Lett. 81, 5362 (1998).

[7] V. Paillard et al., Phys. Rev. Lett. 71, 4170 (1993).

[8] P. Deltour, J.-L. Barrat, and P. Jensen, Phys. Rev. Lett. 78, 4597 (1997).

[9] L. Bardotti et al., Surf. Sci. 367, 276 (1996).

[10] S. Wang, U. Kurpick, and G. Ehrlich, Phys. Rev. Lett. 81, 4923 (1998).

[11] M. Valden, X. Lai, and D. Goodman, Science 281, 1647 (1998).

[12] U. Heiz, A. Sanchez, S. Abbet, and W.-D. Schneider, J. Am. Chem. Soc. 121, 3314 (1999).

[13] I. Goldby, B. von Issendorff, L. Kuipers, and R. Palmer, Rev. Sci. Instrum. 68, 3327 (1997).

[14] S. Hall, M. Nielsen, A. Robinson, and R. Palmer, Rev. Sci. Instrum. 68, 3335 (1997).

[15] H.-P. Cheng and U. Landman, Science 260, 1304 (1993).

[16] K. Bromann et al., Science 274, 956 (1996).

[17] H. Haberland, Z. Insepov, and M. Moseler, Phys. Rev. B 51, 11061 (1995).

[18] H. Haberland et al., in Proceedings of the International Conference on Beam Processing of Advanced Materials, Cleveland, 1995 (unpublished).

[19] G. Betz and W. Husinsky, Nucl. Instrum. Methods Phys. Res., Sect. B 122, 311 (1997).

[20] H. Hsieh, R. Averback, H. Sellers, and C. Flynn, Phys. Rev. B 45, 4417 (1992).

[21] R. Averback, T. D. de la Rubia, H. Hsieh, and R. Benedick, Nucl. Instrum. Methods Phys. Res., Sect. B 59-60, 709 (1991).

[22] R. Smith et al., Nucl. Instrum. Methods Phys. Res., Sect. B 102, 211 (1995).

[23] R. Webb, R. Smith, I. Chakarov, and K. Beardmore, Nucl. Instrum. Methods Phys. Res., Sect. B 112, 99 (1996).

[24] S. Carroll et al., J. Phys. Condens. Matter 8, L617 (1996).

[25] S. Carroll, K. Seeger, and R. Palmer, Appl. Phys. Lett. 72, 305 (1998).

[26] S. Carroll, S. Hall, R. Palmer, and R. Smith, Phys. Rev. Lett. 81, 3715 (1998).

[27] C. Henry, Surf. Sci. Rep. 31, 231 (1998).

[28] D. Brenner, Phys. Rev. B 42, 9458 (1990).

[29] G. Ackland, G. Tichy, V. Vitek, and M. Finnis, Philos. Mag. A 56, 736 (1987).

[30] H. Rafii-Tabar, H. Kamiyama, and M. Cross, Surf. Sci. 385, 187 (1997).

[31] J. F. Ziegler, J. P. Biersack, and U. Littmark, The Stopping and Ranges of Ions in Solids (Pergamon, New York, 1985).

[32] R. Smith, M. Shaw, and R.P. Webb, J. Appl. Phys. 83, 3148 (1998).

[33] R. Smith, Atom and Ion Collisions in Solids and at Surfaces (Cambridge University Press, Cambridge, England, 1997).

[34] S. Hobday and R. Smith, J. Chem. Soc. Faraday Trans. 93, 3919 (1997).

[35] M. Moseler, J. Nordiek, and H. Haberland, Phys. Rev. B 56, 15439 (1997). 